\title{
Custos da produção de madeira de paricá na região de Paragominas, PA
}

\author{
Rafaela da Silveira ${ }^{1}$, Gilson Fernandes da Silva ${ }^{1}$, Daniel Henrique Breda Binoti ${ }^{1}$, Leticia da Paschoa Manhães ${ }^{1 *}$ \\ Anny Francielly Ataide Gonçalves ${ }^{1}$, Mariana de Aquino Aragão ${ }^{1}$
}

${ }^{1}$ Universidade Federal do Espírito Santo, Av. Gov. Lindemberg, 316, Centro, CEP 29550-000, Jerônimo Monteiro, ES, Brasil

\begin{abstract}
"Autor correspondente:
lpmanhaes.3@gmail.com
\end{abstract}

Termos para indexação:

Custos operacionais

Espaçamento

Schizolobium amazonicum

Index terms:

Operating costs

Spacing

Schizolobium amazonicum

Histórico do artigo:

Recebido em 05/10/2017

Aprovado em 18/12/2017

Publicado em 29/12/2017

doi: 10.4336/2017.pfb.37.92.1508
Resumo - A potencialidade silvicultural e econômica do paricá (Schizolobium amazonicum) tem incentivado a expansão desta cultura, principalmente na região norte do país, despertando a necessidade de estudos sobre a espécie. Deste modo, objetivouse neste trabalho estimar a produção de paricá na região de Paragominas, PA, aos 5, 6 e 7 anos, em três classes de produtividade, considerando cinco arranjos de plantas, sendo identificados os principais custos de produção, além dos mais afetados pelos arranjos das plantas. A estimativa volumétrica foi obtida pela modelagem em nível de povoamento e a análise dos custos, por meio da metodologia do custo operacional. Foi possível inferir que o arranjo das plantas $3 \mathrm{~m} \times 2 \mathrm{~m}$ foi superior em volume, diante dos outros arranjos analisados. O custo operacional efetivo representou, em média, $65 \%$ dos custos totais de produção, sendo o transporte o custo individual mais expressivo em todos os arranjos de plantas analisados. A manutenção, dentre o conjunto de atividades, foi a mais significativa no plantio, fato que pode estar relacionado ao número e ao longo período para a execução das atividades. Os menores arranjos das plantas apresentaram os menos custos totais de produção, dependentes ou não da remuneração pelo uso dos capitais permanente e circulante.

\section{Cost of paricá wood production in Paragominas region, Para State, Brazil}

\begin{abstract}
The silvicultural and economic potential of paricá (Schizolobium amazonicum) has encouraged the expansion of this crop, mainly in the northern region of the country, provoking the need for studies on the species. The objective of this study was to estimate paricá production in Paragominas region, Para State, Brazil, at 5,6 and 7 years, in three classes of productivity considering five plant arrangements, being identified the main costs of production, in addition to those most affected by plant arrangements. The volumetric estimation was obtained by modeling in population level and the analysis of the costs, through the methodology of the operational cost. It was possible to infer that the arrangement of plants $3 \mathrm{~m} \mathrm{x} 2 \mathrm{~m}$ was superior in volume, compared to the other analyzed plant arrangements. The effective operating cost represented on average $65 \%$ of the total production costs. Transport was the most significant individual cost, in all analyzed plant arrangements. The maintenance, among the set of activities, was the most significant in planting, fact that may be related to the number and the long period for the activities execution. Smaller plant arrangements presented the higher total costs of production, depending or not on the remuneration for the use of the permanent and current capital.
\end{abstract}




\section{Introdução}

O paricá (Schizolobium amazonicum Huber ex Ducke) é uma espécie heliófila, tolerante a solos de baixa fertilidade, adaptando-se à precipitação média anual superior a $1.600 \mathrm{~mm}$ e temperatura média de $24,8{ }^{\circ} \mathrm{C}$, características típicas da região amazônica (Crespo et al., 1995; Carvalho, 2007). Esta capacidade de adaptação, aliada ao rápido crescimento e produção, vem tornando o paricá uma fonte de renda alternativa, principalmente na região norte do país.

Segundo a Associação Brasileira de Florestas Plantadas (ABRAF) (Anuário..., 2013), em 2012 foram contabilizados 87.901 ha de plantios de paricá, nos estados do Pará, Tocantins e Maranhão. Este montante é resultado da boa aceitação desta espécie no mercado madeireiro, devido aos seus variados fins, como a produção de lâmina e compensado, forros, palitos, papel, móveis, além de peças de acabamento e molduras. Porém, a falta de informações silviculturais e econômicas do paricá têm inviabilizado a expansão de novos plantios em outras regiões do país.

Embora o correto levantamento dos custos de produção seja essencial para o sucesso de qualquer empreendimento, ainda existem muitas dificuldades para a sua obtenção. Vários estudos vêm sendo realizados com o objetivo de identificar os métodos que mais se enquadram à realidade do setor florestal. Entre estes, está o custo operacional, desenvolvido por Matsunaga et al. (1976). Neste método se incluem apenas as despesas efetivamente desembolsadas pelo agricultor, somadas à depreciação de máquinas e equipamentos e mão de obra familiar. A remuneração devida a todos os outros fatores de produção é de responsabilidade do resíduo, ou seja, a diferença entre o custo operacional e o valor de venda (Matsunaga et al., 1976; Conab, 2010).

O êxito nas estimativas de custos de produção agrícola vem estimulando pesquisadores da área florestal a utilizarem esta metodologia também para culturas florestais, principalmente neste momento em que o Brasil depende de medidas estruturais que promovam a redução dos custos de produção e incentivem o investimento, para retomada da competitividade dos produtos nacionais de base florestal no mercado internacional. Assim, objetivou-se neste trabalho identificar os principais custos de produção bem como o efeito dos diferentes arranjos espaciais e classes de produtividade sobre os custos de produção do paricá.

Pesq. flor. bras., Colombo, v. 37, n. 92, p. 597-604, out./dez. 2017

\section{Material e métodos}

Os dados utilizados neste estudo foram originários de povoamentos homogêneos de paricá (Schizolobium amazonicum), plantados sob os arranjos $3 \mathrm{~m} \times 2 \mathrm{~m}, 3 \mathrm{~m}$ x $3 \mathrm{~m}, 4 \mathrm{~m}$ x $3 \mathrm{~m}, 4 \mathrm{~m}$ x $4 \mathrm{~m}$ e $5 \mathrm{~m}$ x $5 \mathrm{~m}$, localizados entre os municípios de Dom Elizeu e Paragominas, região nordeste do Estado do Pará.

Foram inventariados povoamentos de paricá, anualmente, até o quinto ano, sendo analisadas três classes de produtividade com base na altura dominante média em cada arranjo das plantas, a saber: baixa $(17 \mathrm{~m}-19 \mathrm{~m})$; média $(19 \mathrm{~m}-21 \mathrm{~m})$ e alta $(21 \mathrm{~m}$ $24 \mathrm{~m})$. Posteriormente, empregando-se o modelo de crescimento e produção proposto por Silva et al. (2013) aos dados de inventário, os volumes comercial e de lâminas foram estimados para diferentes arranjo das plantas e qualidades de local nas idades de 5, 6 e 7 anos.

A partir de informações sobre as variáveis: área basal por hectare (GHA), índice de sítio (SI), altura dominante (HD) - considerou-se o conceito de Hart, utilizando a altura média das 100 árvores com maior diâmetro a 1,30 $\mathrm{m}$ do solo (DAP) do povoamento - e número de árvores por hectare (NHA) na idade de cinco anos, pode-se projetar a produção para as idades em análise.

Para a obtenção da HD na idade i, número de árvores $\mathrm{ha}^{-1}$ na idade futura e a área basal ha ${ }^{-1}$, foram utilizadas as equações 1, 2 e 3 respectivamente.

$$
S I=\frac{H D_{i}\left(1+\exp \left(\left(1.82404-A_{i}\right) / 0.43105\right)\right)}{1+\exp \left(\left(1.82404-A_{\text {ind }} / 0.43105\right)\right)}
$$

Em que: = base de logaritmo neperiano; $=$ altura dominante na idade $\mathrm{i}$; $=\mathrm{i}$-ésima idade $(\mathrm{i}=5,6,7) ; \mathrm{e}=$ idade índice (5 anos).

$$
N H A_{2}=N H A_{1}-\left(\left(\frac{A R E A}{H D_{1}}\right)^{-2.0848}\left(A_{2}^{2.0356}-A_{1}^{2.0356}\right)\right)
$$

Em que: = número de árvores por hectare na idade futura; = número de árvores por hectare na idade corrente; = área média ocupada por cada planta no plantio; = altura dominante na idade corrente; = idade corrente; $\mathrm{e}=$ idade futura.

$$
G H A_{i}=0.00523 H D_{i}{ }^{1.45720} N H A^{0.53967}{ }_{i}
$$

Em que: $\mathrm{NHAi}=$ número de árvores por hectare na idade $\mathrm{i}$.

Após a obtenção de todas as variáveis, os volumes comercial e de lâminas foram estimados pelas equações 4 e 5 . 


$$
\begin{aligned}
V T H A_{i} & =1.22839 G H A_{i}^{0.93941} H D_{i}^{0,71858} \\
V G H A_{i} & =0.03388 G H A_{i}^{0.69131} H D_{i}^{1.83907}
\end{aligned}
$$

Em que: $V T H A_{i}=$ volume comercial ha' ${ }^{-1}$ e $V C H A_{i}=$ volume de lâminas ha ${ }^{-1}$.

Para análise dos custos de produção florestal foi necessária a coleta de dados anuais de todas as despesas e receitas contraídas, em cada arranjo das plantas, durante o período entre 2006 e 2013.

\section{Análise econômica}

Os custos envolvidos nas atividades de implantação e condução dos povoamentos foram disponibilizados por produtores rurais da região de Paragominas. Empresários locais disponibilizaram os dados referentes à exploração e transporte da madeira até a empresa, referente a uma distância média de $80 \mathrm{~km}$.

Para comparação entre os valores incorridos durante períodos diferentes no tempo, fez-se o deflacionamento dos custos para o período inicial (implantação dos povoamentos). A correção da perda monetária causada pela inflação foi obtida por meio do índice geral de preço - disponibilidade interna (IGP - DI).

Posteriormente, os valores deflacionados foram descapitalizados para o ano 0 (2006), a fim de facilitar a análise econômica (equação 6).

$$
V_{0}=\frac{V_{n}}{(1+i)^{n}}
$$

Em que: $\mathrm{V}_{0}=$ valor inicial correspondente ao período de implantação do povoamento $(\mathrm{R} \$) ; \mathrm{V}_{\mathrm{n}}=$ valor final correspondente ao ano $j(\mathrm{R} \$) ; i=$ taxa de juros (6\% a.a.); e $n$ $=$ número de períodos (anos).

Para o cálculo dos custos de produção do paricá, foi adotada a metodologia descrita pelo Instituto de Economia Agrícola (IEA), órgão vinculado à Secretaria de Agricultura e Abastecimento do Estado de São Paulo (Mello et al., 1976).

Neste caso, consideraram-se os custos efetivos de produção, ou seja, o dinheiro desembolsado pelo proprietário para o pagamento da matéria-prima, produtos ou serviços adquiridos. Para contabilizar o custo operacional efetivo (COE), identificaram-se todas as atividades desde a implantação até a exploração dos povoamentos de paricá e os gastos estimados para obtenção dos custos desembolsados pelo produtor, sendo os custos deflacionados e descapitalizados para 2006.

Não considerou-se a depreciação nesse estudo, devido à indisponibilidade de informações sobre as máquinas, equipamentos e benfeitorias. Assim, considerou-se que todos os maquinários e equipamentos utilizados para a implantação, condução, extração e transporte dos povoamentos são de origem terceirizada.

Os custos de produção foram, então, divididos, segundo sua origem, sendo classificados de acordo com a metodologia de custos proposta pelo IEA, em: custo operacional efetivo (COE) - correspondente a todos os custos explícitos; custo operacional total (COT) - correspondente ao somatório do custo operacional efetivo e mão de obra familiar (MO); e custo total (CT) - soma do custo operacional total e do custo de oportunidade $(\mathrm{CO})$, que é a remuneração do capital circulante e do capital fixo.

Os custos operacional total e total foram obtidos pela aplicação das equações 7 e 8 .

$$
\begin{gathered}
C O T=C O E+M O \\
C T=C O T+C O
\end{gathered}
$$

Em que: $\mathrm{COT}=$ custo operacional total; $\mathrm{COE}=$ custo operacional efetivo; $\mathrm{MO}=$ mão de obra familiar; $\mathrm{CT}=$ custo total; $\mathrm{CO}=$ custo de oportunidade.

\section{Resultados}

\section{Estimativa de volume}

Com base nas equações ajustadas por Silva et al. (2013) para povoamentos de paricá, foi possível estimar o volume comercial e de lâminas, em três idades diferentes e em diferentes classes de produtividade, nos cinco arranjo das plantas, como pode ser observado na Tabela 1. 
Tabela 1. Volumes comercial (VTHA) e de lâminas (VCHA) estimados, nos diferentes arranjos produtivos de plantio de paricá, na região de Paragominas, PA.

\begin{tabular}{|c|c|c|c|c|c|c|c|}
\hline \multirow{3}{*}{ Idade } & \multirow{3}{*}{$\begin{array}{c}\text { Arranjo } \\
\text { (m) }\end{array}$} & \multicolumn{3}{|c|}{ VTHA $\left(m^{3} h^{-1}\right)$} & \multicolumn{3}{|c|}{$\operatorname{VCHA}\left(\mathrm{m}^{3} \mathrm{ha}^{-1}\right)$} \\
\hline & & \multicolumn{6}{|c|}{ Classe de produtividade (m) } \\
\hline & & Baixa & Média & Alta & Baixa & Média & Alta \\
\hline \multirow{5}{*}{$\begin{array}{l}\text { ô } \\
\vdots \\
\text { in }\end{array}$} & $3 \times 2$ & 162,29 & 185,11 & 193,36 & 56,38 & 68,58 & 9,15 \\
\hline & $3 \times 3$ & - & 154,98 & 181,26 & - & 61,63 & 6,71 \\
\hline & $4 \times 3$ & - & 133,28 & 159,83 & - & 54,76 & 0,09 \\
\hline & $4 \times 4$ & 71,33 & 135,51 & 146,23 & 28,00 & 57,05 & 4,68 \\
\hline & $5 \times 5$ & 73,51 & 87,90 & 130,93 & 31,91 & 41,24 & 2,31 \\
\hline \multirow{5}{*}{ 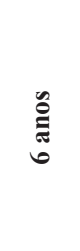 } & $3 \times 2$ & 151,73 & 168,65 & 184,39 & 53,70 & 64,11 & 6,66 \\
\hline & $3 \times 3$ & - & 146,06 & 174,39 & - & 59,12 & 4,73 \\
\hline & $4 \times 3$ & - & 123,25 & 152,44 & - & 51,88 & 7,87 \\
\hline & $4 \times 4$ & 71,78 & 117,45 & 134,10 & 28,27 & 51,46 & 0,82 \\
\hline & $5 \times 5$ & 71,15 & 88,67 & 117,05 & 31,18 & 41,58 & 7,59 \\
\hline \multirow{5}{*}{$\stackrel{\check{E}}{\stackrel{\tilde{\Xi}}{n}}$} & $3 \times 2$ & 143,15 & 156,07 & 164,06 & 51,44 & 60,54 & 0,29 \\
\hline & $3 \times 3$ & - & 139,20 & 164,89 & - & 57,06 & 1,70 \\
\hline & $4 \times 3$ & - & 118,90 & 146,48 & - & 50,52 & 5,90 \\
\hline & $4 \times 4$ & 70,28 & 114,64 & 130,67 & 27,83 & 50,55 & 9,67 \\
\hline & $5 \times 5$ & 70,20 & 87,26 & 115,15 & 30,87 & 41,09 & 6,90 \\
\hline
\end{tabular}

\section{Custos de produção}

Nos povoamentos em estudo, os custos de implantação variaram de $\mathrm{R} \$ 800,00$ a $\mathrm{R} \$ 1.800,00$ entre os arranjos das plantas extremos, sendo menor, quanto menor o número de plantas por hectare. Este montante representa de $18 \%$ a $26 \%$ do custo total de produção do paricá, demonstrando sua importância em relação a outros custos.

O custo da mão de obra foi considerado como um custo operacional efetivo, pois não houve somente um gasto, mas um desembolso, ou seja, uma saída de caixa. A mão de obra familiar foi parte do custo de produção, pois consistiu em uma alocação de recurso produtivo (mão de obra) para a geração do produto (madeira), representando cerca de $10 \%$ dos custos de produção.

O custo total foi obtido pelo custo operacional total acrescido da remuneração paga ao capital investido e ao custo da Terra (Tabela 2).

Os custos de produção da madeira de paricá estão apresentados na Figura 1.

Tabela 2. Custo total (CT) da produção de paricá, em valores de 2006.

\begin{tabular}{|c|c|c|c|c|c|c|c|}
\hline \multirow{3}{*}{ Idade } & \multirow{3}{*}{ Arranjo (m) } & \multicolumn{6}{|c|}{ Classe de produtividade (m) } \\
\hline & & \multicolumn{3}{|c|}{ CT do volume comercial $\left(\mathrm{R} \$ \mathrm{ha}^{-1}\right)$} & \multicolumn{3}{|c|}{ CT do volume de lâminas $\left(\mathrm{R} \$ \mathrm{ha}^{-1}\right)$} \\
\hline & & Baixa & Média & Alta & Baixa & Média & Alta \\
\hline \multirow{5}{*}{$\begin{array}{l}\text { ô } \\
\stackrel{\Xi}{E} \\
\text { in }\end{array}$} & $3 \times 2$ & $7.301,46$ & $7.301,46$ & $7.301,46$ & $9.170,76$ & $9.408,90$ & $9.495,01$ \\
\hline & $3 \times 3$ & - & $6.493,66$ & $6.493,66$ & - & $8.383,41$ & $8.657,76$ \\
\hline & $4 \times 3$ & - & $6.125,27$ & $6.125,27$ & - & $7.790,99$ & $8.068,14$ \\
\hline & $4 \times 4$ & $5.863,08$ & $5.863,08$ & $5.863,08$ & $6.885,22$ & $7.555,23$ & $7.667,13$ \\
\hline & $5 \times 5$ & $5.626,48$ & $5.626,48$ & $5.626,48$ & $6.669,57$ & $6.819,78$ & $7.268,92$ \\
\hline \multirow{5}{*}{ 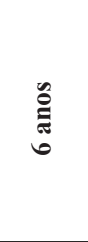 } & $3 \times 2$ & $7.825,37$ & $7.825,37$ & $7.825,37$ & $9.491,33$ & $9.660,26$ & $9.815,26$ \\
\hline & $3 \times 3$ & - & $7.025,82$ & $7.025,82$ & - & $8.638,11$ & $8.917,18$ \\
\hline & $4 \times 3$ & - & $6.659,30$ & $6.659,30$ & - & $8.046,88$ & $8.334,47$ \\
\hline & $4 \times 4$ & $6.405,72$ & $6.405,72$ & $6.405,72$ & $7.283,95$ & $7.736,06$ & $7.900,19$ \\
\hline & $5 \times 5$ & $6.158,36$ & $6.158,36$ & $6.158,36$ & $7.030,43$ & $7.205,23$ & $7.484,83$ \\
\hline \multirow{5}{*}{$\begin{array}{l}0 \\
\stackrel{\Xi}{\sigma} \\
r\end{array}$} & $3 \times 2$ & $8.438,16$ & $8.438,16$ & $8.438,16$ & $9.934,30$ & $10.055,20$ & $10.129,90$ \\
\hline & $3 \times 3$ & - & $7.599,11$ & $7.599,11$ & - & $9.058,37$ & $9.298,60$ \\
\hline & $4 \times 3$ & - & $7.215,28$ & $7.215,28$ & - & $8.484,67$ & $8.742,57$ \\
\hline & $4 \times 4$ & $6.946,69$ & $6.946,69$ & $6.946,69$ & $7.761,32$ & $8.176,20$ & $8.326,18$ \\
\hline & $5 \times 5$ & $6.694,81$ & $6.694,81$ & $6.694,81$ & $7.508,64$ & $7.668,27$ & $7.929,07$ \\
\hline
\end{tabular}




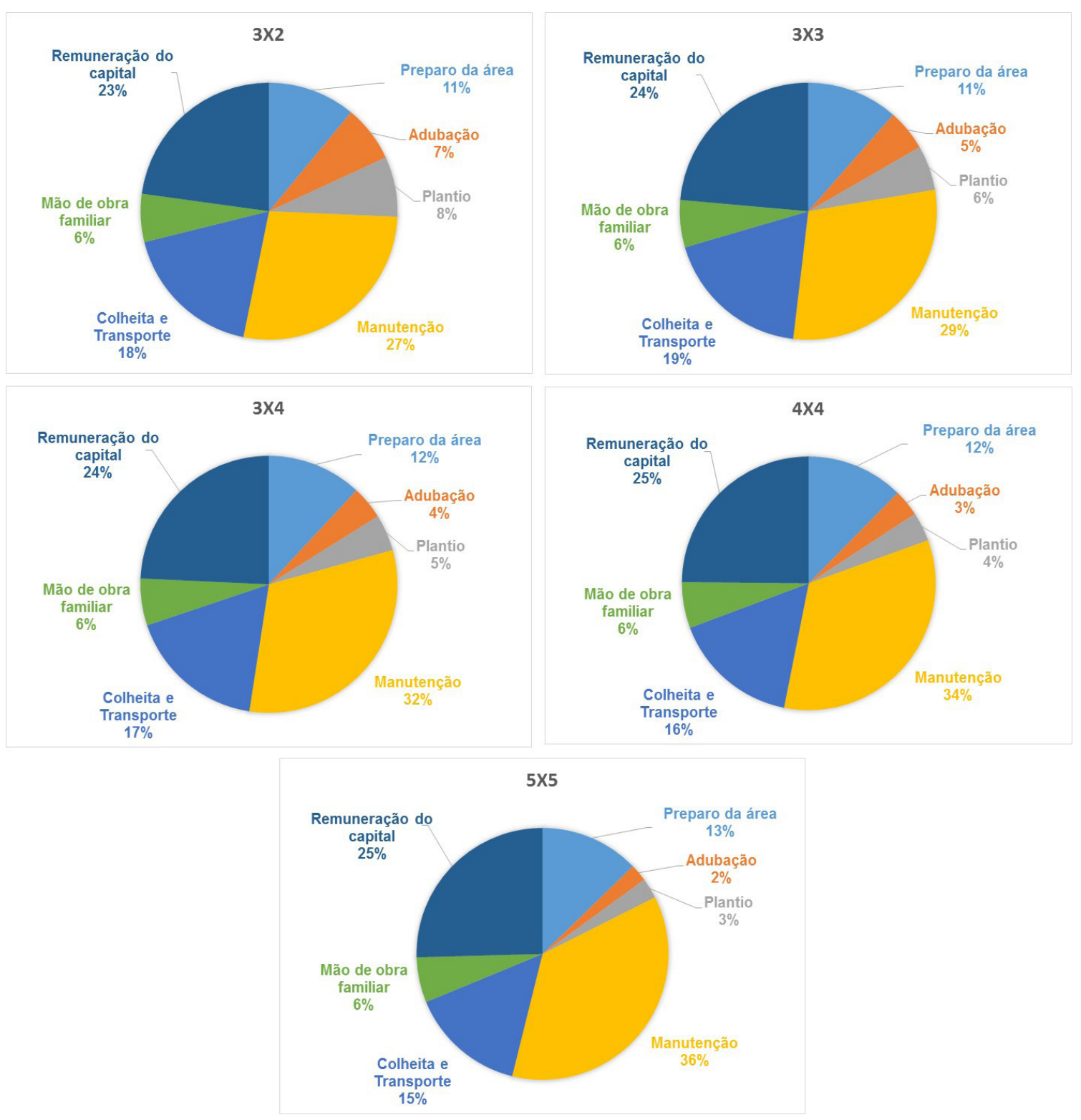

Figura 1. Custos totais da produção de paricá, sob diferentes arranjos das plantas, na região de Paragominas, PA.

\section{Discussão}

\section{Estimativa de volume}

A divisão dos povoamentos em classes de produtividade é explicada por Oliveira et al. (2008). Segundo estes autores, essa estratificação resulta em estimativas de volume mais precisas, próximo à idade de corte, justificando a adoção de três classes.

A falta de informações nos arranjos intermediários das plantas ( $3 \mathrm{~m} \times 3 \mathrm{~m}$ e $4 \mathrm{~m} \times 3 \mathrm{~m}$ ) na classe de produtividade baixa ocorreu pela ausência de povoamentos nestas condições de sítio.

A diferença no volume comercial de madeira foi de $32,3 \%$, entre os arranjos extremos ( $3 \mathrm{~m} \times 2 \mathrm{~m}$ e $5 \mathrm{~m} \mathrm{x}$ $5 \mathrm{~m}$ ), sendo maior o volume nos menores arranjo das plantas. Resultados semelhantes foram encontrados por Macedo et al. (2005), com povoamentos de teca, em Minas Gerais, e Hoffmann (2009), em seu trabalho sobre caracterização dendrométrica e rendimento em laminação de paricá, na região de Paragominas, PA. 
Constatou-se uma diferença de $52,5 \%$ e $54,7 \%$, no volume entre os arranjos extremos, nas classes de produtividade média e baixa, respectivamente. Comportamento semelhante foi observado para o volume de lâminas de paricá. Houve diferença crescente no volume, entre os arranjos extremos, sendo menor na classe de produtividade alta $(21,3 \%)$.

Nas árvores com seis anos de idade, pode-se notar que a diferença no volume entre os arranjos $3 \mathrm{~m} \times 2 \mathrm{~m}$ e $5 \mathrm{~m}$ x $5 \mathrm{~m}$ foi superior à idade de cinco anos. Na classe de produtividade alta, a diferença no volume comercial foi de $36,5 \%$ e o volume de lâminas resultou em diferença de $24,9 \%$.

Nos sítios de baixa produtividade, o volume comercial de madeira no arranjo $3 \mathrm{~m} \times 2 \mathrm{~m}$ foi menor (17,7\%), comparado ao de produtividade alta. Nos arranjos $4 \mathrm{~m} \mathrm{x} 4$ $\mathrm{m}$ e $5 \mathrm{~m}$ x $5 \mathrm{~m}$, esta diferença foi mais significativa, com aproximadamente $46,5 \%$ e $39,2 \%$, respectivamente. Isso se deve, entre outras coisas, à morte natural das plantas com diâmetros maiores, tornando mais expressiva a redução de volume.

Comparando a produção de paricá, em relação à idade de corte, pode-ser observar uma redução no volume de madeira do quinto para o sexto ano. Este comportamento pode ser resultado do autodesbaste, característico da espécie. Por se tratar de uma espécie pioneira, ela atinge o ápice de incremento, na idade média de 5 anos, sendo mais viável seu corte neste período (Carvalho, 2007).

Balloni \& Simões (1980) anteriormente já haviam afirmado que a porcentagem de árvores dominadas e mortas cresce com o avanço da idade, sendo mais intenso e precoce nos arranjos mais apertados. Por isso, a idade de corte deve ser definida em função do ritmo de crescimento, arranjo das plantas, finalidade da madeira e suas interações.

\section{Custos de produção}

O custo no arranjo $5 \mathrm{~m}$ x $5 \mathrm{~m}$ foi semelhante ao encontrado por Silva et al. (2004), para a implantação de 1 ha de eucalipto na região de Brasília, quando os valores foram corrigidos para o mesmo período, demonstrando semelhança na implantação das duas culturas.

Nos povoamentos analisados, não houve diferenças nos custos para implantação e condução dos plantios com classes de produtividade distintas, os quais objetivavam a produção de volume comercial. Em contrapartida, houve o acréscimo de gastos à medida que se reduziram os arranjos do plantio, ou seja, quanto menor a área ocupada por plantas, maiores os gastos com alinhamento, marcação de covas, coveamento, plantio e adubação.

Em relação ao volume de lâminas, ocorreu a distinção dos custos entre classes de produtividade, uma vez que os custos com colheita e transporte estão relacionados com o volume colhido. Assim, em classes de produtividade alta e menor arranjo de plantio, o custo operacional efetivo (COE) foi superior, em comparação aos outros povoamentos analisados.

Aos cinco anos de idade não houve diferença no COE, independente da classe de produtividade, nos povoamentos em que foi mensurado o volume comercial de madeira. Isso foi reflexo da quantidade de plantas inseridas no local, que no plantio foram invariáveis, independente da qualidade do terreno. $\mathrm{O}$ mesmo foi notado quando se estimou os custos para exploração de madeira aos sete anos.

A redução no COE da produção de lâminas com o aumento da idade de exploração é explicada pela redução ocorrida no volume de madeira causado pela mortalidade das plantas, como observado no trabalho de Silva et al. (2013). Assim, diminuem-se proporcionalmente os custos variáveis com colheita, transporte e outras atividades de manutenção.

O custo operacional total (COT) envolveu o COE e a mão de obra familiar, considerada aqui como o custo de administração, ou seja, os proprietários dos povoamentos eram os responsáveis pela administração dos plantios.

O maior custo de administração foi obtido para o arranjo $3 \mathrm{~m} \times 2 \mathrm{~m}$, quando o objetivo era a laminação, com custo de aproximadamente $\mathrm{R} \$ 674,50$. Para o arranjo $5 \mathrm{~m} \times 5 \mathrm{~m}$, com objetivo de venda da floresta em pé, obteve-se o menor custo, com aproximadamente $\mathrm{R} \$ 370,00 \mathrm{ha}^{-1}$.

Resultados semelhantes foram encontrados para a produção de madeira aos seis anos. Os menores custos de administração estão relacionados aos menores custos de implantação e condução dos povoamentos. Aos seis anos, o custo de administração no arranjo $5 \mathrm{~m} \mathrm{x} 5 \mathrm{~m}$, em classe de produtividade alta, com o objetivo de produção de lâminas, foi de aproximadamente $\mathrm{R} \$ 486,60$ e $\mathrm{R} \$$ 385,28 , quando se objetivou a venda da madeira em pé.

Os custos de administração também foram reduzidos à medida que se aumentou o tempo para exploração dos povoamentos, devido a redução do volume por hectare, sendo outro indício de que esta espécie deve ser explorada logo aos 5 anos após o plantio. 
Após contabilizados todos os custos, foi possível inferir que para o volume comercial, em arranjos mais adensados, o custo total foi $22,94 \%$ maior do que nos plantios mais espaçados, independente da classe de produtividade. No entanto, em povoamentos onde o objetivo do plantio era a venda de lâminas, ocorreu uma variação nos custos em fator da classe de produtividade e arranjo de plantio.

A venda da madeira aos seis anos culminou em um acréscimo nos custos de $6,7 \%$ a $8,6 \%$, em comparação com os mesmos povoamentos explorados aos cinco anos. Para a produção de lâminas, o aumento dos custos foi menos expressivo. No arranjo $3 \mathrm{~m} \times 2 \mathrm{~m}$, em classe de produtividade baixa, o aumento foi de 3,4\%.

A exploração de povoamentos aos sete anos foi $13,47 \%$ a 15,96\% maior em relação à exploração aos cinco anos e $7,26 \%$ a $8,01 \%$ para seis anos. Estes números confirmam que os custos têm crescido proporcionalmente com o aumento da idade.

Os custos com a remuneração do capital investido e pelo uso da terra correspondem em média a $24 \%$ dos custos totais de produção. Porém, a maioria dos produtores rurais deixa de contabilizar estes custos por se tratar do pagamento pelo uso dos bens que ele já possui (capital e terra), resultando em valores de produção abaixo do que realmente deve ser computado.

De maneira geral, os custos mais expressivos se referem à manutenção dos plantios, independente do arranjo de plantio adotado (Figura 1). Isso é consequência do maior número de atividades desenvolvidas durante este período, que varia desde o controle de formigas à manutenção de estradas.

Nota-se, no entanto, que quanto maior o arranjo de plantio utilizado, maiores são os custos de manutenção por hectare. Como há menor quantidade de árvores por hectare, a presença de plantas invasoras é mais acentuada, aumentando os custos para seu controle. Em contrapartida, os custos por hectare com adubação, plantio, colheita e transporte diminuem, já que o número de árvores na área é reduzido.

Deve-se ressaltar, ainda, que estes custos podem variar de acordo com a classe de produtividade, a idade em que vão ser explorados os povoamentos e os tipos de atividades empregadas, que podem variar de acordo com o produtor e a região.

Para fins comparativos, utilizando-se a mesma metodologia de custos para a produção de paricá e teca, no arranjo $3 \mathrm{~m}$ x $2 \mathrm{~m}$, observa-se que o custo total para cultivo da teca é de aproximadamente $\mathrm{R} \$ 10.803,09$ (Sanguino, 2009), valor muito próximo ao observado para o plantio de paricá.

\section{Conclusões}

Os custos totais de produção apresentaram diferenças com relação ao espaçamento e classes de produtividade.

O plantio de paricá apresentou, em média, $65 \%$ dos custos totais de produção referentes ao custo operacional efetivo, sendo o transporte o custo individual mais expressivo, para todos os arranjos de plantas analisados. A manutenção apresentou destaque dentre o conjunto de atividades mais significativo nos povoamentos, devido ao número e ao longo período para execução das atividades.

\section{Referências}

Anuário Estatístico da ABRAF 2013: ano base 2012. Brasília, DF: ABRAF, 2013. 148 p. Disponível em: < http://www.abraflor.org.br/ estatisticas/ABRAF13/ABRAF13.BR. pdf>. Acesso em: 19 ago. 2013.

Balloni, E. A. \& Simões, J. W. O espaçamento de plantio e suas implicações silviculturais. IPEF, v. 1. n. 3. p. 1-16, 1980. (IPEF. Série técnica).

Carvalho, P. E. R. Paricá (Schizolobium amazonicum). Colombo: Embrapa Florestas, 2007. 8 p. (Embrapa Florestas. Circular técnica, 142).

Conab (Brasil). Custo de produção agrícola: a metodologia da Conab. Brasília, DF, 2010. 60 p.

Crespo, T. R. et al. Evaluación de algunas leguminosas en el trópico de Cochabamba, Bolivia. In: Evans, D. O. \& Szott, L. T. (Ed.). Nitrogen fixing trees for acid soils: proceedings of a workshop. Morrilton: NFTA / Winroch International, 1995. p. 103-112.

Hoffmann, R. G. Caracterização dendrométrica e avaliação do rendimento em laminação de madeira em plantios de paricá (Schizolobium amazonicum Huber ex. Ducke) na região de Paragominas, PA. 2009. 97 f. Dissertação (Mestrado em Ciências Florestais) - Universidade Federal do Espírito Santo, Jerônimo Monteiro.

Macedo, R. L. G et al. Desenvolvimento inicial de Tectona grandis L.F. (teca) em diferentes espaçamentos no município de Paracatu, MG. Cerne, v. 11, n. 1, p. 61-69, 2005.

Matsunaga, M. et al. Metodologia de custo de produção utilizada pelo IEA. Agricultura em São Paulo, v. 23, p. 123-139, 1976.

Mello, M. et al. Metodologia de custo utilizada pelo IEA. Agricultura em São Paulo, v. 23, p. 123-139, 1976.

Oliveira, M. L. R. et al. Classificação da capacidade produtiva de povoamentos não desbastados de clones de eucalipto. Pesquisa Agropecuária Brasileira, v. 43, n. 11, p. 1559-1567, 2008. 
Sanguino, A. C. Custos de implantação e rentabilidade econômica de povoamentos florestais com teca no estado do Pará. Ciências Agrárias, n. 52, p. 61-78, 2009.

Silva, G. F. et al. Modeling growth and yield of Schizolobium amazonicum under different spacings. International Journal of Forestry Research, v. 2013, p. 10, 2013.
Silva, K. R. et al. Custos e rendimentos operacionais de um plantio de eucalipto em região de cerrado. Revista Árvore, v. 28, n. 3, p. 361-366, 2004. 